\title{
Medios nativos digitales en América Latina: agenda, sostenimiento e influencia
}

Digital Native Media in Latin America: Agenda, Sustainability and Influence

Mídias digitais nativos na América Latina: Uma Agenda, apoio e influência

Jimena ZULUAGA TRUJILLO, Colombia

Universidad de los Andes Colombia / jizuluag@uniandes.edu.co

Silvia Marcela GÓMEZ MONTERO, Colombia

Universidad de los Andes Colombia / sm.gomez1o@uniandes.edu.co

Chasqui. Revista Latinoamericana de Comunicación

N. ${ }^{o} 141$, agosto - noviembre 2019 (Sección Ensayo, pp. 301-316)

ISSN 1390-1079 / e-ISSN 1390-924X

Ecuador: CIESPAL

Recibido: 28-08-2017 / Aprobado: 16-11-2019 


\title{
Resumen
}

Internet cambió para siempre los medios de comunicación. El espacio digital permitió nuevas formas de generar contenidos periodísticos. Los primeros en saltar al ruedo fueron los medios tradicionales, quienes lanzaron sus ediciones en línea. Poco después surgieron nuevos medios, los llamados nativos digitales. En América Latina, factores como la brecha de conectividad y la concentración de la propiedad han influido en el desarrollo del ecosistema digital. Este texto explora tendencias de los nativos digitales latinoamericanos desde tres ejes: las agendas informativas, los modelos de sostenimiento y los efectos de los medios. Es una reflexión sobre la forma en que las iniciativas periodísticas locales han aprovechado las cualidades de internet para hacerse un lugar en la esfera pública.

Palabras clave: periodismo digital; nativo digital; modelo de negocio; internet

\begin{abstract}
Internet changed media forever. The web allowed new ways of generating journalistic content. Legacy media went online first. Shortly after, new media emerged: the so-called digital natives. In Latin America, the connectivity gap and ownership concentration of media are among the factors that have influenced the development of the digital ecosystem. This text explores Latin American digital media from three axes: agenda, business models, and the effects of the media. It is a map of journalistic initiatives that have taken advantage of the characteristics of the Internet to claim a place in the public sphere.
\end{abstract}

Keywords: digital Journalism; Digital Native; business model; Internet

\section{Resumo}

Internet mudou para sempre a mídia. O espaço digital permitiu novas formas de gerarconteúdodenotícias.Oprimeiroasaltar para oanelfosseamídiatradicional, que lançou suas edições online. Pouco depois de nova mídia surgiu o chamado nativos digitais. Na América Latina, fatores como a distância conectividade e a concentração da propriedade influenciaram o desenvolvimento do ecossistema digital. Este artigo explora as tendências de nativos digitais latino-americanos de três áreas: agendas de informação, modelos de apoio e os efeitos da mídia. É uma reflexão sobre algumas iniciativas jornalísticas que se aproveitaram das qualidades da internet para obter um lugar na esfera pública.

Palavras-chave: jornalismo digital; nativos digitais; modelo de negócio; Internet 


\section{Introducción}

Internet puso a tambalear, de manera casi súbita, un modelo de trabajo, sostenimiento y relación con el poder y las audiencias relativamente estable para los medios de comunicación durante 300 años. Su popularización marcó un desafío respecto, por supuesto, a la tecnología y los dispositivos, pero también a los hábitos de consumo. Fue un cambio rápido en lo primero y una transición gradual en lo segundo. Los primeros medios digitales fueron las versiones en línea de los diarios impresos. Pronto la tendencia fue la diversificación, que impuso no solo reutilizar contenidos, también recombinar y recrear, es decir, complementar el impreso mediante la personalización de contenidos y publicidad, agregación, archivos de noticias, y lo que para la época era una novedad: contenidos generados por los usuarios (CGU o UGC en inglés) (Boczkowski, 2006).

La idea de una audiencia pasiva terminó por desdibujarse de una vez por todas. "En la era de internet todos pueden hacerlo todo. Todos pueden ser a la vez editores, autores y periodistas. Todos pueden participar, todos pueden ganar dinero. Ese es el mantra" (Schirrmacher, 2012, p. 1). El nacimiento de los prosumidores (audiencias productoras y consumidoras), desplaza el eje de la comunicación de medios de uno muy vertical hacia uno más horizontal. Manuel Castells (2001) llama esto la autocomunicación de masas, un escenario en el que la autonomía se refuerza a partir de las redes de comunicación horizontales al interior de las cuales los emisores-destinatarios se convierten en el centro de la galaxia internet. Es el aparente fin del monopolio de la información por parte de los periodistas en tanto que ya no es exclusiva de su oficio la posibilidad de contar: "de la comunicación de masas dirigida a una audiencia, hemos pasado a una audiencia activa que forja su significado comparando su experiencia con los flujos unidireccionales de la información que recibe" (p. 184).

En este contexto surgieron los primeros nativos digitales, medios que no tienen un correlato previo en el impreso, la radio o la televisión. En un contexto amplio, uno de los ejemplos más representativos y exitosos es The Huffington Post, que nació en Nueva York en 2005 por iniciativa de Ariana Huffington y Jonah Peretti. El HuffPost, junto a otros como Buzz Feed, mantuvieron, en general, la fórmula de combinar la tradición impresa de la narrativa lineal con, cada vez más, el potencial del hipertexto, la interacción y el formato multimedia (Boczkowski, 2006). Estos emprendimientos establecieron redes de colaboración en las que muchos de los autores trabajaban gratuitamente, exploraron la idea de los contenidos virales y se dedicaron a interactuar y conocer a sus audiencias estableciendo conversaciones y monitoreando al detalle su tráfico (Shappiro, 2012). Esta tendencia se caracteriza por innovar y tomar riesgos, entre otras cosas, porque hay menos qué perder. En América Latina, el surgimiento y desarrollo de los nativos digitales encuentra sus variaciones en el marco de sus particularidades como región en vía de desarrollo político, económico, social 
y tecnológico. Estas condiciones determinarán las agendas, alternativas de sostenimiento y potencial de influencia de los medios digitales.

\section{Los pioneros y el reto de la conectividad}

Entre 1994 y 1996 muchos periódicos de América Latina lanzaron sus ediciones web. Durante buena parte de las dos décadas siguientes, los protagonistas del ecosistema fueron en su mayoría las versiones en línea de los llamados medios tradicionales. Entre 2010 y 2014 se creó el $73 \%$ de los medios nativos digitales (Meléndez, 2016, p. 10). En Nicaragua y República Dominicana estos fueron, incluso, la primera forma de periodismo en internet. Es preciso anotar que muchos de estos medios nacientes eligieron la web como alternativa ante su incapacidad económica de funcionar en versiones impresas. Además de las ventajas que ofrece la tecnología en términos de potencial de alcance y bajos costos de operación, la actividad periodística digital en la región se ha apoyado también en academia y los grupos de investigación, las Facultades de Comunicación de las universidades y la unión de interesados, mayormente intelectuales (Salaverría, 2016).

Uno de los pioneros latinos fue El Faro, en El Salvador, fundado en 1998 por el periodista Carlos Dada y el empresario Jorge Simán. Empezó como un medio en internet que más tarde se imprimiría; pero esto último no sucedió. Su idea era aprovechar la infraestructura tecnológica para competir con la oferta local. Funcionaba como una red de corresponsales internacionales a la que más tarde se sumaron jóvenes voluntarios que estudiaban periodismo. Se financiaron durante mucho tiempo con fondos provenientes de cooperación hasta que tuvieron capacidad para pagar salarios. En 2014, su equipo estaba compuesto por 22 personas (Salaverría, 2016, p. 152) y ya eran financieramente autónomos.

En Argentina, por su parte, Interlink Headlines News, Rosarionet y Periodismo.com, publicaron desde los años noventa como nativos web. Asimismo, la región fue prolífica en blogs. En Brasil, entre 2003 y 2006 hubo un pico de surgimiento de blogs; sin embargo, muchos desaparecían porque el desafío más grande era siempre encontrar un modelo de sostenimiento estable. En Colombia, el directorio Blogalaxia cuenta más de 5.400 blogs de humor, cocina, farándula (Salaverría, 2016, p. 85). Uno de los portales más consultados y calificado como uno de los que ejerce mayor influencia en este país es La Silla Vacía (2009), cuyos contenidos están concentrados en el poder y la política. La Silla se ha sostenido gracias al apoyo de fundaciones, donaciones de los usuarios, ingresos de publicidad y cursos dictados por el medio. Este modelo mixto, al que le apuestan otros medios no es, sin embargo, una fórmula infalible ni necesariamente estable. Una iniciativa importante para hacer frente a este asunto, apoyarse entre medios y permanecer conectados como red es el grupo de los ALiados, conformado en 2013 por diez nativos de nueve países con el objetivo de compartir estrategias de financiación y experiencias de éxito 
para seguir haciendo periodismo independiente y de calidad ${ }^{~}$. Otros medios, varios de ellos aún más jóvenes que los pioneros, como 14ymedio, de Cuba, El mostrador, de Chile, o Sudestada, de Ecuador, han fortalecido recientemente su voz en la esfera pública regional (Meléndez, 2016).

Si bien su crecimiento es evidente, el relativamente nuevo ecosistema de los digitales de la región enfrenta varios retos. Uno relacionado con un asunto de infraestructura y a la vez político: la brecha de conectividad. Según el Índice de desarrollo de las tecnologías de la información y las comunicaciones de 2016, los latinoamericanos con mejor puntaje son, en su orden, Uruguay, Argentina y Chile, que ocupan los puestos 47, 55 y 56 en el ranking mundial. Los más rezagados son Honduras, Nicaragua y Cuba, en el 126, 131 y 135, de una lista de 175 (International Telecommunication Union, 2016). La brecha digital en América Latina es menor que en Asia y África que ocupa los últimos lugares, salvo algunas excepciones como Sudáfrica y países insulares pequeños que están por encima del número 100. El caso de Asia es interesante porque si bien un país grande como Rusia ocupa el puesto 43 (por encima de todos los latinoamericanos) el continente representa el 4.0\% de la población total de internautas en el mundo, versus el 60\% de la población que habita en la región. América Latina representa el 10\% del total de usuarios de internet en el mundo (Comscore, 2015) y esta cifra es similar al porcentaje que representa la región dentro del total de la población mundial: el 8,6\% (Worldometers, 2015). En Norteamérica y Europa la proporción es contraria: estas dos regiones aportan el $41 \%$ de internautas, aunque solo representan el 15,4\% de la población mundial.

El otro asunto importante es la brecha entre las zonas urbanas y rurales. El caso colombiano es elocuente: mientras en Bogotá la penetración de internet es del 20,4\%, en la zona de los llanos orientales mayormente rural, departamentos de Vichada, Guainía y Vaupés, es del o.2\% (Ministerio de Tecnologías de la Información y las Comunicaciones de Colombia, MinTic, 2016). El informe Ciberperiodismo en Iberoamérica confirma que esta es una situación común en la región:

Disponer de una conectividad razonable ha sido un privilegio del que, durante buena parte de estos veinte años, han podido gozar únicamente los habitantes de los países con economías más potentes y los habitantes de grandes núcleos urbanos (...) el acceso rural aumenta globalmente mucho más despacio que el acceso urbano, de modo que la conexión de banda ancha en los hogares de zonas rurales sigue siendo una tarea pendiente (Salaverría, 2016, p. 24).

1 ALiados está integrado por: Cipery The Clinic (Chile); Confidencial (Nicaragua); El Faro (El Salvador); IDL-Reporteros (Perú); Plaza Pública (Guatemala); Puercoespín (Argentina); La Silla Vacía (Colombia); Animal político (México); y Agencia pública (Brasil). La Silla Vacía (6 de junio de 2013). Nace alianza latinoamericana de medios como La Silla. Recuperado de https://goo.gl/5Q6vaj y Animal Político (20 de junio de 2013). Nace ALiados, red de medios digitales de América Latina. Recuperado de https://goo.gl/yGfjSE 
Estas condiciones, por supuesto, condicionan el periodismo digital en tanto restringen sus audiencias y, en ese sentido, su alcance y potencial de impacto.

\section{Apuesta por agendas independientes}

Un segundo reto de los nativos digitales es la concentración de la propiedad de los medios, que en América Latina es altísima (Becerra y Mastrini, 2010). Algunos nativos han asumido la bandera de la independencia como contrapoder, casi como una militancia por posicionar temas que son, en su criterio, relevantes para lo público, pero ausentes o sin suficiente cobertura en las agendas tradicionales. En palabras de Daniel Moreno, director de Animal Político de México:

¿Por qué pasarse a un medio digital? Por la necesidad de estar en un medio en el que te reconozcas. (...) Los medios tradicionales en México básicamente son reproductores de declaraciones oficiales, defensores del status quo y, en esa lógica, el nicho que nos dejan abierto es gigantesco (participación en conversatorio Construyendo una democracia efectiva: el rol de los medios de comunicación. The Carter Center y Universidad de los Andes. Bogotá, 7 de noviembre, 2014)

Enrique Naveda, editor general de Plaza Pública de Guatemala, coincide en esta vocación de diversificar los temas a partir del criterio editorial:

Hemos tenido cierto éxito en re-tematizar la agenda. Introducir muchos temas que antes no estaban en la parrilla (...). Por ejemplo, el racismo, la discriminación, temas de género, la organización social popular aparecían esporádicamente y como disfunciones, anécdotas de la vida diaria, cuando en realidad son las cosas que están moldeando el futuro del país. (...) La relación entre la élite económica y la gente de este país y el gobierno y la organización judicial y el congreso es prácticamente no un tema tabú porque hay voces que lo mencionan, pero no es agenda de los medios (comunicación personal, 8 de julio, 2014).

Sin embargo, la agenda propia significa en muchas ocasiones alejarse de los temas tendencia que son los que más generan audiencia, es decir, visitas, uno de los indicadores determinantes para persuadir anunciantes e incluso demostrar influencia. En general, de recibir dinero.

Slant News fue un emprendimiento digital fundado en 2015 por periodistas con experiencia en medios como The Huffington Post y The New York Daily News, cuyo modelo de pago a los periodistas consistía en una compensación mensual fija baja y un bono variable con base en los clics que recibieran sus historias (Murtha, 2015). Este modelo no es nuevo para los negocios digitales. Pero, al tratarse de un sitio de noticias, ilustra un debate de los medios informativos, más antiguo que internet, sobre quién pone la agenda: el interés comercial o el interés público. 
Distanciados del modelo de medios como BuzzFeed, varios de los nativos digitales de la región son radicales en su convicción de no caer en la llamada dictadura del clic. Así lo explica Daniel Moreno, de Animal Político:

A mí me interesan las visitas, por supuesto. Pero creo que es importante que las visitas no determinen tu contenido. (...) No somos el sitio más visitado de México y no queremos serlo. Cuando yo veo a mis competidores enloquecidos publicando notas sin un mínimo de rigor, me digo: hasta allá no llegamos. (...) Queremos que nos lean, pero basándonos en el periodismo en el que creemos (participación en conversatorio Construyendo una democracia efectiva: el rol de los medios de comunicación. The Carter Center y Universidad de los Andes. Bogotá, 7 de noviembre, 2014)

Plaza Pública, La Silla Vacía y Animal Político tienen en común que sus fuentes de ingresos están diversificadas, es decir, no viven solo de la pauta publicitaria, por lo que jugársela por la agenda independiente es menos riesgoso que para los tradicionales en términos financieros.

En cuanto al contenido, llama la atención que, ante la inmediatez de internet, varios de los nativos han apostado precisamente por lo contrario: el periodismo reposado y la investigación a profundidad. El $35 \%$ de los evaluados en el Primer Estudio de Medios Digitales y Periodismo en América Latina hace periodismo de investigación (Meléndez, 2016, p. 10). De modo que hay una tendencia por apostar a la generación de contenidos con un cuidadoso proceso de verificación y de rigor respecto a las fuentes en una "obsesión con el poder y los poderosos" (Lozano y León, 2013, p. 5). Un periodismo con propósitos políticos, que tiene una misión como vigía del poder para señalar y visibilizar irregularidades, pero, además, que se traza como objetivo principal la transformación de la sociedad.

Mónica González, directora deCiper, deChile, asegura que esnecesario "atacar los temas que provocan miedo a la sociedad (...) [y que] a la mano privada nunca se la investiga" (Ruiz, 2013, p. 12). González abandonó los medios tradicionales por su proyecto propio. Carlos Fernando Chamorro, director de Confidencial de Nicaragua, dice que en los países en donde impera la impunidad y "los Estados no le rinden cuentas a los ciudadanos muchas veces a los periodistas nos toca ir más allá de la mera misión de simplemente informar"(Aristegui, 2015, p. 1). El periodismo independiente, en su concepto, debe asumir el riesgo de incomodar al poder tradicional. En América Latina algunos periodistas independientes son perseguidos, amenazados, censurados e, incluso, desprestigiados. La bloguera cubana Yoani Sánchez, cuyo trabajo periodístico se opone al régimen, fue víctima de bloqueo y sus usuarios eran redirigidos a un sitio en el que la difamaban (BBC, 2014). A pesar de esto, los líderes de medios encuentran en el espacio digital una oportunidad de independencia en términos de agenda. Esto también se lo permite su forma de financiarse. De este modo, afinan su sentido político como lugares de contrapoder y militancia. Martín Rodríguez Pellecer, 
fundador de Nómada de Guatemala, antes director de Plaza Pública, sostiene que el rigor y la profundidad rinden sus frutos:

Tenemos 120.000 visitas al mes, más o menos 80.000 usuarios únicos, y entre estos muchos urbanos de clase media y, sobre todo, tomadores de decisión, dentro y fuera del país. Nuestra influencia todavía es muy reducida, pero al menos hacemos que quienes tienen poder tengan que leernos, para enterarse mejor del país o para saber si escribimos sobre ellos (Peñaloza, 2013, p. 5)

En cuanto a las narrativas, el formato dominante en los nativos es el texto, lo cual se explica en clave de los recursos económicos y humanos disponibles para la producción de contenido. No obstante, en varios medios hay ejemplos de propuestas narrativas multimedia, como el proyecto Meninas em Jogo (Chicas en juego) desarrollado por Agencia Pública de Brasil, un reportaje en formato de cómic sobre la explotación sexual de niñas y jóvenes en el estado de Ceará, en el contexto del Mundial de Fútbol de 2014; La puerta giratoria del poder, de Ciper (Chile) y Quién es quién de La Silla Vacía (Colombia), visualizaciones interactivas sobre las relaciones entre los poderosos del país. Otra tendencia interesante tiene que ver con la verificación de información, como lo hace el medio Chequeado (Argentina) y la sección Detector de mentiras de La Silla Vacía. Ante la sobreabundancia de información en la red, la legitimidad puede estar en la verificación de la información falsa, más que en la primicia.

\section{Sostenibilidad: la pregunta clave}

Un cambio fundamental definió el futuro de la prensa en el siglo XVIII: la pauta publicitaria. El momento en que los diarios se convirtieron en un medio, no solamente para difundir ideas e información, si no para vender otros productos. El modelo de negocio que se configuró se mantuvo durante más de dos siglos en los que la prensa vivió de la pauta comercial y de la venta de diarios, dos entradas por una salida: el impreso. A finales del siglo XX este modelo flaqueó. Si bien los medios digitales no necesitan grandes inversiones, los dividendos, al igual que las inversiones, son modestos, al menos para la mayoría. Y la venta de publicidad no es el único y seguro camino para la sostenibilidad en internet. El único consenso que parece haber hasta el momento es que nada está inventado aún. Las formas de sostenimiento de los medios digitales en la región, diversas por demás, pueden clasificarse en cuatro grandes categorías:

1. Pauta publicitaria. Aunque es la fuente principal, en internet no es igual de rentable. Primero, porque la pauta es mucho más barata; segundo, porque el mercado ha tardado tiempo en tomarla en serio, afirman Olga Lucía Lozano y Juanita León (2013), fundadoras de La Silla Vacía:

Las barreras de entrada para acceder a la pauta de las grandes empresas que anuncian sus productos en medios digitales es alta. Por un lado, los medios 
tradicionales con frecuencia usan la pauta digital como una napa en los paquetes publicitarios de sus impresos (...). Por otro, las centrales de medios que administran esta pauta tienen unos incentivos -a veces perversos-, para redirigir el dinero de los anunciantes hacia donde reciben mayores comisiones y no necesariamente donde están las audiencias mejor segmentadas (p. 68).

Sin embargo, la pauta digital se sofistica cada vez más. Las cantidad de información que se puede recoger acerca de la audiencia hace que las posibilidades de segmentación crezcan, haciéndola más atractiva. De la muestra de 34 nativos consultados por el Primer Estudio de Medios Digitales y Periodismo en América Latina el 38\% aseguró apostarle exclusivamente a la publicidad para su sostenimiento (Meléndez, 2016, p.13). Esto puede obedecer bien a que hay confianza en que el modelo de pauta digital se estabilizará, o bien dar cuenta del peso de la tradición al apostar por la forma de financiación clásica.

2. Suscripciones y pagos de los usuarios. Una de las maneras más comunes de recibirlos es la barrera de pago o paywall: el contenido gratuito es limitado y para acceder a contenidos de más calidad o extensión el usuario debe pagar. Estos pagos pueden ser por cada contenido, suscripciones anuales o mensuales. Una versión sofisticada es el crowdfunding o financiación colectiva que consiste en recibir dinero (a manera de donaciones), sin que eso signifique necesariamente pagar por el contenido pues no hay barrera de pago. La Silla Vacía es uno de los pioneros en intentar este modelo a través de su campaña anual Súper amigos, lanzada en 2012, que en 2016 recaudó 87,5 millones de pesos colombianos, unos 30 mil dólares (León, 2016, p. 1). Otros medios como Animal Político (México) y Ojo Público (Perú) han replicado esta estrategia con campañas similares. A finales de 2014. Animal Político lanzó Amigos de Animal, que en 2016 se volvió permanente y ha recibido en donaciones cerca de dos mil dólares por mes desde julio de 2016 (Donadora, 2016).

3. Becas, premios, apoyos y subsidios de otras entidades. En octubre de 2013 Jeff Bezos, fundador del portal Amazon, decidió comprar The Washington Post, uno de los periódicos estadounidenses con más trayectoria. Comprarlo significó prácticamente salvarlo de la quiebra (Filloux, 2013). La movida de Bezos es metafórica de una de las formas de sostenimiento de los medios en línea: la filantropía. Aunque este no es precisamente un modelo de negocio, las becas, premios y subsidios de entidades internacionales se han convertido en la forma de sostenimiento de muchos emprendimientos periodísticos en el mundo y en la región la tendencia es clara: de los 34 medios presentes en el Primer Estudio de Medios Digitales y Periodismo en América Latina el 19\% dijo contar con financiación de cooperación internacional (Meléndez, 2016, p. 13). Entre 
otros, Agencia Pública (Brasil), Chequeado (Argentina), Ciper (Chile), El Faro (El Salvador), Ojo Público (Perú) y Nómada (Guatemala), cuentan entre sus ingresos becas y aportes de entidades internacionales como Open Society Foundations y la Fundación Ford.

4. Diversificación. El informe de innovación del New York Times, filtrado en mayo de 2014, menciona los eventos, conferencias y festivales como formas de financiación alternativa (Benton, 2014). Medios tradicionales colombianos como Semana y El Tiempo entraron ya hace tiempo en esta tendencia. Además de los eventos, algunos medios periodísticos venden asesorías, talleres de alfabetización digital o publicaciones de contenido comercial para pagar la operación del medio informativo. Un caso interesante es El Faro (El Salvador) que montó una tienda en línea donde vende libros, música, películas, y piezas de arte y diseño.

Muchos emprendimientos periodísticos de América Latina le apuestan a modelos híbridos, es decir, que combinan varias fuentes. De todos modos, cabe anotar que de los 34 medios nativos digitales reseñados en el Primer Estudio de Medios Digitales y Periodismo en América Latina una vasta mayoría (85\%), inició el proyecto sin pensar antes en el modelo de negocio, aun cuando 20 de los 34. son empresas o sociedades con ánimo de lucro (Meléndez, 2016). De lo anterior, resulta lógico que el financiamiento y sostenibilidad a largo plazo sea lo que identifican como su principal preocupación.

\section{Alcance, impacto e influencia: a quién le hablan y quién los oye}

Las métricas de los medios de comunicación han estado asociadas al alcance del mensaje en términos cuantitativos: el tiraje de un diario o el rating de un noticiero, por ejemplo. Esto se explica porque la mayoría de estos parámetros de medición nacieron en la industria de la publicidad: estiman el tamaño de las audiencias y esto es un parámetro de qué tan atractivas son para los anunciantes.

La naturaleza de internet hace que el camino que cada usuario traza en la red sea fácilmente identificable. Prácticamente hay registro de todo y todo se puede medir. Por lo mismo, además del número de visitas, los medios saben muchas más cosas de sus audiencias digitales: cuánto tiempo permanecen en el sitio, cuántos contenidos leen en promedio, qué visitan antes y después. Sin embargo, esta información es solo una parte de la respuesta a una pregunta más compleja acerca de los efectos que pueden tener los contenidos de los medios en los votantes o en los tomadores de decisiones públicas (Schiffrin y Zuckerman, 2015).

$\mathrm{Al}$ igual que sucede con los medios tradicionales, la mayoría de las métricas de audiencias en internet dan pocas luces del impacto social de los mensajes noticiosos o de los medios mismos. Así lo explica Paul F. Lagunes, profesor de la Universidad de Columbia, Estados Unidos: 
Si monitoreáramos el efecto de 100 noticias sobre el comportamiento de la gente y notáramos que no hay ninguna diferencia, podríamos concluir que no hay impacto mediático. Pero puede pasar que la historia número 101 haga que la gente salga a protestar a la calle. Tal vez esa historia tocó una fibra sensible, o tal vez las historias 99, 100 y 101 tuvieron un efecto de bola de nieve que llevó a la movilización. (...) Y está también el asunto de los cambios que no se ven. Una noticia puede modificar la forma en que vemos el mundo sin que eso signifique que tomemos acción inmediatamente (Schiffrin y Zuckerman, 2015, p. 13).

Es preciso señalar la relevancia que han adquirido las métricas de impacto e influencia de los contenidos informativos en el contexto digital, especialmente por el hecho de que muchos medios nativos (y también tradicionales) tienen una importante fuente de financiación en agencias de filantropía que, a cambio de su apoyo, piden evidencia del impacto social del emprendimiento que financian. Esto es algo común en la industria privada y en otras causas de beneficencia, pero relativamente ajeno a las métricas de los medios. Es incluso complejo el consenso respecto de los términos mismos para estos indicadores, que pueden agruparse someramente en tres categorías: alcance, influencia e impacto (Schiffrin y Zuckerman, 2015).

El alcance hace referencia fundamentalmente a las métricas cuantitativas tradicionales: cuántas personas se involucran con un contenido o serie de contenidos (diarios vendidos o, para el caso digital, páginas vistas, visitantes únicos, contenidos compartidos en redes, entre otros). El problema de estas métricas es que los contenidos más vistos o más compartidos no son necesariamente los de mejor calidad periodística o mayor potencial de impacto público: son generalmente las historias inspiradoras (llamadas en inglés feelgood stories), como videos que se hacen virales en redes. Y en este sentido, son métricas relativamente fáciles de complacer, pues es viable guiarse por las cifras de lo que llama más clics o más actividad en redes para crear contenido similar y aumentar el tráfico. En general, cualquier medio sabe qué temas o personajes de la agenda tienen este efecto de carnada de clics (clickbait en inglés). "Generar tráfico es lo más fácil. Uno siempre está debatiéndose entre tener influencia o tener tráfico masivo. (...) Yo tengo clarísimo qué genera tráfico y qué no, incluso en política, sin llegar a la caricatura de poner videos de gaticos”, dice la directora de La Silla Vacía, Juanita León (participación en conversatorio Construyendo una democracia efectiva: el rol de los medios de comunicación. The Carter Center y Universidad de los Andes. Bogotá, 7 de noviembre, 2014).

El segundo tipo de indicadores, los de influencia, tratan de medir la manera en que los contenidos informativos afectan el diálogo público; cómo puede una historia afectar la conversación pública o cómo la cobertura de un asunto noticioso desde determinado ángulo cambia o determina la forma en que ciudadanos y organizaciones lo ven. Esta reflexión no surge con internet. Mucho de lo investigado en influencia de medios nace de la teoría de establecimiento de temas (en inglés agenda setting) esbozada por Walter Lippmann a principios 
del siglo XX, desarrollada por Bernard Cohen, Maxwell McCombs y Donald Shaw en los años sesenta y setenta, y ampliamente abordada en los estudios de comunicación. En general se han hecho estudios de caso particulares (en periodos electorales o sobre representación de personajes en los medios en determinados contextos, por ejemplo) pero la medición cualitativa de la influencia escapa aun las formas cuantificables de medición de medios.

Finalmente, las métricas relacionadas con el impacto buscan establecer cómo el contenido ayuda o genera un cambio de política o un movimiento social. El periodista norteamericano Jonathan Stray (2010) se refiere al impacto en este sentido como el problema de la última milla: ¿cómo hacer que la cobertura mediática tenga impacto social? Hay casos excepcionales en los que el impacto de una historia o la cobertura prolongada de una historia es nítido. Tal vez el ejemplo por excelencia sea el caso Watergate en Estados Unidos y más recientemente la investigación del diario The Boston Globe sobre pederastia en la iglesia católica, que fue incluso llevada al cine. Un ejemplo local puede ser lo sucedido en Colombia en 2005, cuando Claudia López (investigadora y analista, hoy senadora) y periodistas de Semana.com, de forma paralela y luego de manera conjunta, documentaron una serie de atipias electorales en las zonas de influencia paramilitar que desencadenaron el escándalo mediático y luego las investigaciones judiciales y condenas a congresistas y líderes políticos dentro de la llamada Parapolítica (alianzas de políticos con grupos armados ilegales de derecha, llamados paramilitares).

En Estados Unidos, las unidades investigativas de algunos medios hacen seguimiento del impacto de su trabajo a partir, por ejemplo, del monitoreo de los editoriales y piezas de opinión que citan su trabajo o de cambios en políticas públicas en los asuntos específicos de sus historias. El impacto es, tal vez, el tipo de indicador más potente pero a su vez más difícil de cuantificar, entre otros, porque la investigación en ciencias sociales ha demostrado que el cambio social, por lo general, sucede en el transcurso de décadas y que la cobertura de medios tiene incidencia solo cuando se combina con fuerzas sociales que tienen lugar de manera paralela (Schiffrin y Zuckerman, 2015).

En América Latina, hay seguimiento a indicadores de alcance con diversos grados de detalle entre los medios y exploraciones aún escasas en cuanto a mediciones de impacto e influencia. En términos de alcance, los nativos digitales están todavía rezagados respecto de las versiones digitales de los tradicionales. El caso de Colombia es elocuente: según datos estimados de SimilarWeb, mientras ElTiempo.com, versión digital del diario impreso con más circulación en el país, tiene el 6\% del tráfico de los sitios de noticias consultados en Colombia, Pulzo.com, el nativo digital más popular, alcanza solo el $0.9 \%, \mathrm{Y}$ de los diez sitios de noticias más consultados del país solo dos son nativos (Martínez y Zuluaga, 2016, p. 22). El caso de México es similar, confirma Rodrigo Gómez García profesor investigador de la Universidad Autónoma Metropolitana (cuestionario electrónico, 2 de marzo, 2016). 
Respecto de la influencia e impacto en la región, expertos coinciden en que los indicadores pueden relacionarse con factores como generar reacción en líderes de opinión, influenciar la agenda misma de medios y, en general, su relevancia para la opinión pública: cuantificar, por ejemplo, la frecuencia con que otros medios o líderes de opinión los citan o reaccionan a sus contenidos, explica María Elena Gronemeyer, profesora investigadora de la Universidad Católica de Chile (cuestionario electrónico, 24 de noviembre, 2015). Sobre los digitales en particular, la influencia se ve además en la generación de eco en los medios tradicionales; esto, al tiempo, es un factor de reputación, explica María Paula Martínez, profesora investigadora de la Universidad de los Andes de Bogotá (cuestionario electrónico, 25 de noviembre, 2015). Este indicador cobra sentido considerando, además, la brecha de alcance entre digitales tradicionales y nativos.

Martín Becerra, profesor investigador de la Universidad Nacional de Quilmes (Argentina), anota al respecto que el tamaño de la audiencia (alcance) no es el único factor determinante en términos de impacto, pues "hay también medios muy influyentes con audiencias no masivas, porque interpelan a líderes de opinión pública, al estamento político, económico o jurídico" (cuestionario electrónico, 10 de diciembre, 2016). La Silla Vacía es uno de ellos pues, aunque según datos estimados de SimilarWeb es superado en alcance por otros 25 medios (Martínez y Zuluaga, 2016, p. 50), es el cuarto sitio de noticias más consultado entre los líderes de opinión del país (después de las páginas de $E l$ Tiempo, Semana y El Espectador, todos medios tradicionales), y el segundo entre líderes de Bogotá, solo superado por ElTiempo.com (Cifras y Conceptos, 2016).

Dado el alcance limitado, es común en varios países del continente la percepción de que el impacto de los medios nativos digitales es aún marginal en comparación con el de sus contrapartes tradicionales y en particular con las versiones digitales de los medios tradicionales. Como señala María Elena Gronemeyer, en Chile, uno de los países de la región con más alto índice de conectividad, "hay pocos medios digitales nativos muy influyentes. Generalmente lo son más las versiones digitales de los medios convencionales" (cuestionario electrónico, 24 de noviembre, 2015). Pesa que los nativos digitales son jóvenes y la reputación se construye en el tiempo.

Así pues, el potencial de influencia de los nativos digitales de la región parece estar más relacionado con el impacto sobre nichos más pequeños pero poderosos, que con un gran alcance. Juanita León, directora de La Silla Vacía lo confirma, al menos para el caso de este medio:

Por ejemplo: un día que nos alegra a nosotros es el día que subimos la hoja de vida de Miguel Pinedo, hijo de un parapolítico, y que iban a nombrar viceministro, y tres horas después de que lo sube La Silla, lo bajan de la página de Presidencia y deciden no nombrarlo porque no tenía ningún mérito para estar ahí (participación en conversatorio Construyendo una democracia efectiva: el rol de los medios 
de comunicación. The Carter Center y Universidad de los Andes. Bogotá, 7 de noviembre, 2014).

Llama la atención que en el caso de Chile, Argentina, México y Colombia los expertos coinciden en que los nativos han hecho investigaciones y denuncias que han desencadenado escándalos mediáticos y públicos recogidos después por otros medios. María Elena Gronemeyer destaca un ejemplo de Chile:

El Mostrador ha revelado casos de corrupción y escándalos políticos y ha publicado columnas más provocativas que los medios convencionales, además siendo muy crítico de todo el espectro político y social. Esos contenidos han marcado pauta en los medios y han marcado el debate público (cuestionario electrónico, 24 de noviembre, 2014).

Para Víctor Solano, analista de medios colombiano, la potencial y creciente influencia de los nativos digitales reside precisamente en uno de los ejes analizados en este texto y que resulta un elemento común de muchos nativos digitales: la independencia de sus agendas (cuestionario electrónico, 1 de marzo, 2016).

\section{Conclusión}

Las dinámicas propias de internet transformaron la interacción de los medios de comunicación con las audiencias y las formas de generación de contenidos. Los nativos digitales han aprovechado la oportunidad de difusión en la red para hacerse a una agenda independiente como factor de identidad, llevando por bandera las cualidades del buen periodismo, uno que logre retar al poder y desafiar las barreras de la dependencia comercial, uno de investigación y análisis. Lo anterior, sin embargo, en medio de una instalada incertidumbre sobre su sostenibilidad financiera, frente a la cual se han explorado estrategias diversas que les han permitido continuar en la batalla. A esto se suma que los nativos no parecen registrar en cifras un alto nivel de consumo masivo; pero sí dentro de nichos específicos. No obstante su alcance limitado, los nativos digitales alcanzan cada vez mayores índices de influencia e impacto en esferas de opinión y, con ello, de participación como agente de poder o, por lo menos, de vigilancia.

\section{Referencias bibliográficas}

Aristegui, C. (16 de octubre de 2015). En países donde prevalece la impunidad, los periodistas hacemos las investigaciones. Aristegui Noticias. Recuperado de https://goo.gl/Kv7Fp2

BBC. (21 de mayo de 2014). Bloquean en Cuba la nueva revista digital de Yoani Sánchez. BBC. Recuperado de https://goo.gl/J3T1jy 
Becerra, M. y Mastrini, G. (2010). Concentración de los medios en América Latina: Tendencias de un nuevo siglo. Contratexto (18). 41-64.

Benton, J. (15 de mayo de 2014). The leaked New York Times innovation report is one of the key documents of this media age. Nieman Lab. Recuperado de https://goo.gl/W5Kxbl

Boczkowski, P. (2006). Digitalizar las noticias. Buenos Aires, Argentina: Manantial.

Castells, M. (2001). La galaxia internet. Barcelona, España: Plaza y Janés.

Cifras y Conceptos (2016). Panel de opinión 2016. Recuperado de https://goo.gl/569cKA

Comscore. (2015). Global Digital Future in Focus. Recuperado de https://goo.gl/tNJpiq

Donadora. (2016). \#ElijoAnimal. Fondeo de periodismo independiente. Donadora.

Recuperado de https://goo.gl/yYrmEi

Filloux, F. (septiembre 23 2013). How Jeff Bezos can save the Washington Post. The Guardian. Recuperado de https://goo.gl/CD5B6L

Gómez-Rodulfo, M. (13 de junio de 2013). Entrevista a Carlos Dada. Más investigación. Recuperado de https://goo.gl/rL5N7X

International Telecommunication Union. (2016). Information and Communications Technologies, ICTs, Development Index 2016. Recuperado de https://goo.gl/BHz5bH

Lozano, O.L. y León, J., (2013). La Silla Vacía, un modelo del periodismo del futuro. Bogotá, Colombia: eCiseros Libros y OSF.

León, J. (9 de diciembre de 2016). ¡Gracias Súper Amigos!. La Silla Vacía. Recuperado de https://goo.gl/dMpYFE

Martínez, M. y Zuluaga, J. (2016). Ocho claves para entender las audiencias digitales en Colombia. Bogotá, Colombia: Ceper y FNPI. Recuperado de https://goo.gl/dtuwJi

Ministerio de Tecnologías de la Información y las Comunicaciones de Colombia, MinTic (2016). Boletín trimestral de las TIC. Cuarto trimestre 2015. Recuperado de https://goo. $\mathrm{gl} / 22 \mathrm{KQ}_{4} \mathrm{M}$

Meléndez, J. (2016). Primer Estudio de Medios Digitales y Periodismo en América Latina. México D.F., México: Factual. Recuperado de https://goo.gl/XoGGNr

Murtha, J. (13 de abril de 2015). What it's like to get paid for clicks. Columbia Journalism Review. Recuperado de https://goo.gl/YgqZrK

Peñaloza, P.P. (23 de marzo de 2013). Entrevista a Martín Rodríguez Pellecer. Más investigación. Recuperado de https://goo.gl/7uy36F

Ruiz, I. (20 de mayo de 2013). Entrevista a Mónica González. Más Investigación. Recuperado de https://goo.gl/mwrnBl

Salaverría, R. (ed.) (2016). Ciberperiodismo en Iberoamérica. Madrid, España: Fundación Telefónica y Editorial Ariel. Recuperado de https://goo.gl/Mqx7nz

Schiffrin, A., y Zuckerman, E. (2015). Can We Measure Media Impact? . Stanford Social Innovation Review, 13 (4). Stanford University. Recuperado de https://goo.gl/v7FGRN

Schirmacher, F. (2012). Las promesas incumplidas de Internet. El Malpensante (137). Recuperado de https://goo.gl/R7gFrx

Shappiro, M. (2012). Six degrees of aggregation. Columbia Journalism Review (mayo - junio 2012). Recuperado de https://goo.gl/RcsksZ

Stray, J. (15 de diciembre de 2010). Does Journalism Work? . Jonathan Stray. Recuperado de https://goo.gl/4,cAaXj

Wordometers (2015). World Population by Region. Recuperado de https://goo.gl/vvkPc4. 
